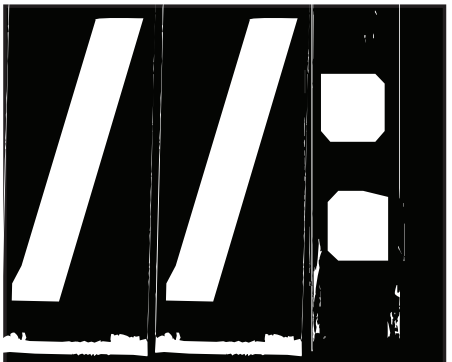

aduca"ción y cōmunicación

18: 61-72 Mayo 2019

\section{LA FUENTE DE LAS DESIGUALDADES EN LAS REDACGIONES PERIODÍSTICAS. VOCES FEMINISTAS CONTRA LA DISGRIMINACIÓN Y LA VIOLENCIA DE GÉNERO EN LOS MEDIOS}

\section{The source of inequalities in journalistic writing. feminist voices against discrimination and gender violence in the media}

Florencia Laura Rovetto

Investigadora CONICET. Centro de Investigaciones Feministas y Estudios de Género

(CIFEG)

Facultad de Ciencia Política y RRII. Universidad Nacional de Rosario (UNR), Santa Fe,

Argentina.

Investigadora CONICET. Doctora en Periodismo y Ciencias de la Comunicación y Magister en Comunicación y Educación por la Universidad Autónoma de Barcelona. Licenciada en Comunicación Social por la Universidad Nacional de Rosario. Directora del Centro de Investigaciones Feministas y Estudios de Género (CIFEG) de la Facultad de Ciencia

Política y Relaciones Internaciones (UNR).

Integrante de la colectiva "Ni Una Menos" de la ciudad de Rosario.

E.mail: florencia.rovetto@cifegunr.com

Lucía Anahí Figueroa

Becaria CONICET. Centro de Investigaciones Feministas y Estudios de Género (CIFEG). Facultad de Ciencia Política y RRII. Universidad Nacional de Rosario (UNR) Rosario,

Santa Fe, Argentina.

Licenciada en Comunicación Social.

Integrante del CIFEG (Centro de Investigaciones Feministas y Estudios de Género). Miembro del Centro de Investigación en Mediatizaciones (CIM) de la UNR. E.mail: lulafigueroa87@cifegunr.com 


\section{II: Feminismo y medios de comunicación}

\section{Resumen}

Esta comunicación se desprende de un proyecto de investigación más amplio centrado en el estudio de la división sexual del trabajo en las redacciones periodísticas. Aquí proponemos reflexionar sobre los escenarios de posibilidad que existen en la prensa local para la inclusión de la perspectiva de género en el tratamiento de las noticias. Tomando como objeto de indagación el problema de las voces autorizadas y la utilización de las fuentes en los tres periódicos impresos que se editan en la ciudad de Rosario (Argentina) nos preguntamos por los modos en que la división sexual del trabajo opera en las redacciones y afecta la calidad de la producción informativa. Este ejercicio, a su vez, pretende iluminar el problema de las desigualdades de género en las prácticas periodísticas en un contexto caracterizado por una creciente movilización política y cultural feminista que ubica a los medios de comunicación en la mira de sus demandas y reivindicaciones.

Palabras clave: feminismos, periodismo, género, fuentes.

Abstract:

This communication emerges from a broader research project focused on the study of sexual division at journalism. Here we propose to reflect on the possibilities for inclusion of the gender perspective in the treatment of news. Taking as an object of inquiry the problem of authorized voices, and the use of sources at three printed newspapers of Rosario (Argentina), we ask ourselves about the ways in which the sexual division works in newsrooms by affecting the quality of information contents. This exercise, in turn, aims to illuminate the problem of gender inequalities in journalistic practices, in a context characterized by a growing feminist political and cultural mobilization that places the media in the sights of their demands.

Key words: feminisms, journalism, gender, sources.

Recibido 26-01-2019 / Revisado 19-02-2019 / Aceptado 24-03-2019 / Publicado 01-05-2019 


\section{Contexto de indagación}

$\mathrm{A}^{\prime}$ ntes de adentrarnos en el ejercicio analítico propuesto, centrado en indagar el uso androcéntrico de las fuentes en la prensa informativa local, nos interesa describir sucintamente el contexto en el que situamos este ejercicio empírico, porque es en relación con un particular clima de época, determinado por una serie de acontecimientos específicos, que el análisis propuesto cobra especial sentido.

El año 2018 quedará marcado a fuego en la memoria del movimiento feminista argentino por haber logrado instalar en la escena pública sus históricas demandas $\mathrm{y}$, fundamentalmente, por hacerlas masivas, alcanzando mayor adhesión política en sus reivindicaciones. Pero, a su vez, por haber puesto en la mira con agudeza y profundidad los modos con los que opera el sistema patriarcal en tanto régimen de violencia e impunidad sostenido en todos los espacios de la vida en comunidad: tanto en ámbitos públicos como privados, el estado, los medios de comunicación, los espacios de trabajos y educativos, etc.

La memoria colectiva recordará que el año 2018 estuvo atravesado por un arco temporal que se inicia en el mes de enero, con la presencia de reconocidas feministas locales (periodistas, comunicadoras e investigadoras) invitadas durante varias semanas al "living" del programa de televisión "Intrusos"(1) para explicar qué es el feminismo, cómo opera el patriarcado, qué son los micromachismos o por qué "el aborto sigue siendo una deuda de la democracia"; y se cierra el 11 de diciembre con la denuncia pública de la colectiva Actrices Argentinas al actor Juan Darthés por violar a la actriz Thelma Fardín, en 2009, -cuando ella tenía 16 años- durante una gira del elenco de la telenovela "Patito feo" en Nicaragua (2).
Estos dos acontecimientos, ubicados en cada extremo de un año calendario, irrumpen en la escena mediática e invitan a considerar tres elementos, indisolublemente articulados entre sí, que aquí postulamos a modo de hipótesis de trabajo para orientar los objetivos de esta indagación. Tales elementos pueden ser considerados como síntomas emergentes de una época profundamente interpelada por los saberes y prácticas feministas, que ubican a los medios de comunicación en la mira y nos permiten postular que:

La falta de paridad en las redacciones y la escasa presencia de mujeres en los espacios de toma de decisión pone al descubierto el entramado ideológico de la desigualdad y la división sexual del trabajo en los medios de comunicación.

La denuncia pública de las violencias sexistas atraviesa todos los sectores y se visibiliza en los medios de comunicación como escenario de disputa y circulación de nuevos sentidos.

La ausencia de perspectiva de género en la producción, abordaje y tratamiento de los contenidos informativos es, además de una resultante de lo anterior, la expresión simbólica del sexismo en el discurso informativo y el uso de fuentes funciona como un indicador de la discriminación de género en las redacciones.

A partir de aquí, en los siguientes apartados recorremos distintas dimensiones para problematizar la complejidad de este fenómeno en el campo de estudio propuesto. Comenzamos por la caracterización de los 
condicionantes materiales y simbólicos del trabajo en las redacciones informativas. En segundo lugar, procuramos relacionar los debates sobre las desigualdades de género en el acceso a los medios de comunicación con las luchas actuales del movimiento feminista que cuestionan la discriminación de género en todos los ámbitos de la vida y ubican a las violencias sexistas como la principal barrera para el desarrollo humano basado en la universalidad de todos los derechos. Finalmente, exponemos los resultados del análisis del uso androcéntrico de las fuentes, tomando lo publicado en una jornada informativa por tres periódicos locales, para ilustrar de qué manera la discriminación de género evidencia el calado profundo de la violencia simbólica que los medios ejercen cotidianamente.

\section{Diagnóstico de la desigualdad en las redacciones periodísticas}

Las políticas institucionales, la organización laboral, la línea editorial, la gestión sindical y hasta la administración comercial o estatal de los medios periodísticos están atravesadas por las desigualdades de género, con evidentes efectos políticos y simbólicos en las rutinas profesionales y sus productos. La discriminación se despliega más en el desarrollo profesional que en el acceso, habida cuenta de que las mujeres son mayoría en las redacciones (Balseiro Expósito, 2008). La segregación sexo-generizada se despliega cotidianamente en las redacciones reafirma y legitima el androcentrismo en los medios de comunicación (Moreno Sardà, 1998).

Amén de todos los desarrollos teóricos y evidencias empíricas que han demostrado y constatado la persistencia de la mirada androcéntrica en los discursos informativos, en tanto la perspectiva de quienes tradi- cionalmente detentan el poder (3), el androcentrismo también se expresa en los modos de organizar los vínculos laborales y en las propias dinámicas de trabajo (4).

Es innegable que el actual escenario social y político habilita debates y discusiones que ponen en la mira dichas desigualdades en los medios de comunicación, en tanto espacios privilegiados para su reproducción -hacia afuera y hacia adentro-. De ahí que, tal como afirma Lakoff, "poder expresar lo que está ocurriendo puede cambiar lo que está ocurriendo, cuando menos a la larga" (2006: 111). Esta interpelación también es referida por Segato (2013) cuando señala que al haberle puesto nombre a la discriminación de género en los medios de comunicación se ha generado la legitimación de una mirada crítica que renueva la sensibilidad ética de la sociedad en este y otros aspectos problemáticos y complejos que reclamaban un lugar en la agenda pública y una reforma del entendimiento de la cultura occidental.

Ciertamente, en los últimos años y en distintas partes del mundo, las periodistas se vienen organizando local y regionalmente, visibilizando sus reivindicaciones y demandas específicas en manifiestos públicos. Por ejemplo, en España, a principios de marzo de 2018 en el marco de la organización de la Huelga Internacional de Mujeres (\#8M), y bajo el lema «Las periodistas paramos», se inició una convocatoria que en pocos días logró el apoyo de más de 8 mil mujeres profesionales de los medios de comunicación, y promovió un manifiesto en el que se denuncian todas las formas de desigualdades que afectan cotidianamente a las mujeres y a otras personas feminizadas en el sector. También animaron a colectivos de periodistas de otros países a realizar su propio manifiesto, conscientes de la "relevancia social" del trabajo que desempeñan y 
preocupadas por:“(...) la visión parcial de la realidad que tantas veces ofrecen los medios y en la que falta la presencia y aportaciones de las mujeres. El feminismo también es necesario para mejorar el periodismo" [5]. También, a este lado del Atlántico, el acontecimiento conocido como \#NiUnaMenos que irrumpe públicamente, convocando a la movilización del 3 de junio de 2015 con la consigna \#BastaDeFemicidios, es promovido por periodistas y comunicadoras que, junto con organizaciones feministas, logran instalar estas problemáticas en la agenda pública como nunca antes. Si bien volveremos sobre este acontecimiento en el siguiente apartado, la potencia del grito de \#NiUnaMenos, que rápidamente se hace global (de Titto, 2018), radicó en las estrategias de visibilización cada vez más creativas y certeras dirigidas a disputar sentidos sobre la complejidad de las violencias sexistas, interpelando todas las instituciones políticas y sociales, los ámbitos de trabajo y reproducción de la vida, así como los modos hegemónicos de representación mediática de las violencias sexistas, ancladas en la “espectacularización” y la repetición de imaginarios victimizantes (Rovetto, 2015).

Ciertamente, después de cuatro convocatorias de \#NiUnaMenos (3 de junio de 2015, 2016, 2017 y 2018), una jornada de huelga de Mujeres (realizada el 19 de octubre de 2016 tras el cruel asesinato de la joven Lucía Pérez) y las dos Huelgas Internacionales (\#8M) llevadas adelante hasta ahora, la emergencia pública y masiva de la discursividad feminista ha ensanchado sus horizontes políticos, permitiendo desvelar la crisis global del capitalismo heteropatriarcal en su versión neoliberal, así como su impacto en la vida de las mujeres y otro sectores subalternos [6].

Asimismo, lo que todas estas manifestaciones también vienen evidenciando, de forma cada vez más categórica, es el problema de la división sexual del trabajo (Pateman, 2005), que se expresa mediante mecanismos como la segregación laboral horizontal y vertical, el techo de cristal, la brecha salarial (7), como formas en que las violencias sexistas operan solapadas o explícitamente en las organizaciones laborales. Dichas formas fraguan cotidianamente "las relaciones sociales de sexos, comprendidas como relaciones desiguales, jerarquizadas, asimétricas o antagónicas de explotación entre dos categorías de sexos construidas socialmente" (Hirata, 1997: 54).

En cuanto a la segregación laboral basada en el género, por ejemplo, a pesar del significativo aumento de la participación femenina en el mercado de trabajo, sobre todo a partir de la década del 70 y su profundización durante los 90 (Wainerman, 1996), se sostiene que el modo de inserción para las mujeres continúa siendo desigual, sustentado en una segregación horizontal por rama de actividad y una vertical por jerarquía (Rojo Brizuela y Tumini, 2008).

A su vez, los estereotipos de género que asocian a las mujeres con determinadas características se trasladan al mercado de trabajo en virtud de una mayor participación en áreas "blandas", de servicios, de cuidados o vinculadas a tareas reproductivas. Así también, los atributos valorados negativamente son una fuente de limitación a la hora de acceder a cargos de jerarquía que implican atributos menos "empáticos" o de jefatura y control asociados a los varones. Esta barrera invisible que opera en la posibilidad de las mujeres de ocupar lugares de mando fue denominada techo de cristal, (8) porque limita material y subjetivamente su ascenso laboral a los cargos ejecutivos. Para el caso específico del trabajo periodístico se puede constatar que, en la mayoría de las organizaciones, las mujeres son "la tropa" de las redacciones, cumpliendo con el 
sintagma que afirma que "el poder de los medios no está en el terreno, sino en los puestos de responsabilidad de las redacciones" (Rastrollo y Gómez Torralbo, 2006: 148) [9].

Respecto de la segregación vertical las evidencias muestran que con igual educación o experiencia las mujeres sufren discriminación laboral respecto de sus pares varones en todas las áreas (Wainerman, 1996). En el sector de la comunicación y el periodismo esta tendencia se recrudece, ya que, aunque las mujeres tengan los mismos o mayores niveles de educación las oportunidades de acceso y promoción para varones y mujeres son diferentes.

A su vez, en los últimos años se ha puesto de relieve la feminización ciertas profesiones, como el periodismo, a partir del crecimiento de la matrícula femenina en las carreras formación técnica y de grado. Sin embargo, este proceso lejos de garantizar la participación paritaria ha generado mayor precarización en los espacios de trabajo, fomentada por proceso globales de ajuste y flexibilización laboral (Henry, 2013). Es decir que, aun cuando en las redacciones, la presencia de las mujeres haya crecido cuantitativamente respecto a décadas pasadas, su entrada al mercado periodístico coincide con la aplicación global de medidas macroeconómicas que precarizan las condiciones de trabajo, aumentan el desempleo o fomentan formas de subcontratación -part-time ofree-lance-con su consecuente pérdida de derechos laborales y rebaja salarial (Rovetto, 2013).

Por otra parte, en Argentina, el 15\% de las notas firmadas corresponden a autorías de mujeres, según las últimas cifras presentadas por Proyecto de Monitoreo Mundial de Medios (2015) (GMMP, por sus siglas en inglés). De acuerdo a este informe, que se hace cada cinco años, la Argentina tiene el promedio de firmas más bajas de América Latina. El diagnóstico del GMMP determinó que los varones son la "voz experta" en los informativos. La brecha de género en los medios no es solo un síntoma regional ni se cierra a pesar de que las mujeres están cada vez más organizadas y visibles en la agenda pública y política. Solo el $37 \%$ de las informaciones publicadas en los medios tradicionales el año pasado fueron elaboradas por mujeres, a pesar de que éstas copan las universidades de periodismo y comunicación social (GMM, 2015).

Estas cifras, así como la breve caracterización que hemos hecho hasta aquí, exponiendo el entramado ideológico de la desigualdad y los modos en que la división sexual del trabajo atraviesa los medios de comunicación, pone en evidencia que el "tiempo por sí solo" no cambia ni transforma los condicionantes estructurales que imposibilitan la equidad entre varones, mujeres y personas feminizadas en los escenarios de producción de contenidos informativos. Para lograrlo, hacen falta políticas públicas concretas, normativas acordes que las impulsen y regulen su aplicación en las organizaciones públicas y privadas de comunicación, así como medidas afirmativas que fomenten la participación paritaria en todos los cargos y funciones, entre otros imperativos urgentes si se quiere modificar el actual estado de situación.

Pero también es necesario que se profundicen los niveles de movilización social y debate público en torno a las desigualdades de género en todos los ámbitos como sólo el feminismo lo viene impulsando, tal como veremos en el siguiente apartado, donde procuramos sistematizar algunos elementos que expresan las resistencias e interpelaciones que ubican a los medios de comunicación como escenario privilegiado para tal disputa política y cultural. 


\section{Violencias sexistas en primera plana}

Si consideramos los desarrollos teóricos y las estrategias políticas feministas impulsadas en las últimas décadas, como un gran marco interpretativo con capacidad de transformación social, podemos comprender el calado profundo de algunas acciones orientadas a interpelar y socavar el sistema de dominación heteropatriarcal, racista y clasista, y reconocer su capacidad para ampliar derechos, invocando sentidos inclusivos de ciudadanía y proponiendo un nuevo orden civilizatorio basado en nuevos pactos entre mujeres y con otros sectores sociales oprimidos (Femenías, 2002; Lagarde, 2015).

Como señalamos más arriba, los últimos años -en un proceso que fue extendiéndose inclusive continentalmente- estuvieron marcados por las movilizaciones masivas de \#NiUnaMenos y diversas acciones públicas que provocaron la visibilización de persistentes luchas y una gran sensibilización social contra las violencias sexistas. No es casual que esta "oleada" (10) se propague de la mano de comunicadoras, periodistas, artistas, escritoras y activistas jóvenes que tejen redes físicas y virtuales, disputando visiones de mundo y construyendo narrativas contrahegemónicas (De Titto, 2018).

En un plano más declarativo, también la IV Conferencia Mundial sobre la Mujer de Beijing -específicamente, el "Capítulo J" de su Plataforma de Acción- ya en el año 1995, había puesto sobre la mesa del debate político internacional que las desigualdades de género en el acceso muy limitado de las mujeres a los medios de comunicación y a las tecnologías de información, en términos de representación y de empleo, así como su escasa participación como propietarias y producto- ras en los medios de comunicación son poderosas barreras que dificultan la universalidad de los derechos humanos (ONU, 1995).

Algunos de los principios emanados de aquella Conferencia encontraron asidero material en la elaboración y promoción leyes fundamentales en distintos países latinoamericanos, entre las cuales destacamos las denominadas “de segunda generación” (11), relacionadas con el abordaje de las violencias sexistas y las que se orientaron a democratizar y/o desmonopolizar los sistemas de medios de comunicación, tales como la Ley 26.485 de Protección Integral para Prevenir, Sancionar y Erradicar la Violencia contra las Mujeres en los Ámbitos en que Desarrollen sus Relaciones Interpersonales y la Ley 26.522 de Servicios de Comunicación Audiovisual.

A su vez, entre los antecedentes más relevantes, vinculados a la temática abordada, se pueden mencionar las articulaciones de periodistas con visión de género que, a su vez, cuentan con alianzas como la Red Internacional de Periodistas con Visión de Género (RIPVG), fundada en el año 2005 y a Periodistas Argentina en Red (PAR), fundada en el año 2006. Esta última se propuso incidir en la agenda mediática local con la creación del Decálogo para el tratamiento periodístico de la violencia contra las mujeres (2008) y el Decálogo para el tratamiento periodístico de la trata y la explotación sexual (2012), el desarrollo de campañas contra la violencia mediática (2013) y la promoción de la perspectiva de género en las carreras universitarias de periodismo y comunicación social.

En este clima de época situamos la disputa que llevan adelante las periodistas y comunicadoras con perspectiva de género que desarrollan su profesión en medios tradicionales o alternativos, cooperativos y autoges- 
tionados, en un contexto de alta precariedad laboral y ajuste en el sector. El término perio-feministas nos ha servido para nombrar a estas profesionales, en su mayoría jóvenes, que se definen por su identidad laboral como periodistas y se auto-perciben como feministas haciendo de sus prácticas un ejercicio profesional y militante al mismo tiempo. En otras publicaciones hemos observado que su tarea se torna crucial tanto hacia adentro, como hacia afuera de los medios de comunicación (Rovetto y Figueroa, 2018).

Esta convergencia virtuosa contribuye a aumentar las posibilidades de producción y de circulación de sentidos que, hasta el presente, eran vistos como marginales y excluidos de las lógicas hegemónicas propias de los discursos dominantes de circulación masiva. La indispensable articulación entre feminismos y comunicación de discursos contrahegemónicos en torno a elementos socialmente significantes y cruciales en nuestras realidades contemporáneas -como las violencias sexistas, el aborto, las desigualdades producidas por la división sexual del trabajo en todos los ámbitos o la heteronormatividad obligatoria- emerge en discursividades que pulsan por transformar también las prácticas de producción periodísticas, para romper con tabúes sociales y con la normalización mediática de expresiones de sexismo y discriminación.

Las demandas y las reivindicaciones históricas impulsadas por las mujeres en el sector son recurrentes porque se topan, sistemáticamente, con la impermeabilidad de las empresas de comunicación, sus administradores y otros varones periodistas. Así, la desoída necesidad de incorporar la perspectiva de género en las redacciones, la ausencia de medidas que favorezcan la "real" igualdad de oportunidades entre varones y mujeres, las lógicas que reproducen la cultura machista en las redacciones, el acoso u otras formas de violencia sexistas que garantizan su impunidad, son los condicionantes más cuestionados por las nuevas generaciones de mujeres que se incorporan a la profesión periodística.

En la actualidad, la función de los medios de comunicación como espacios de democratización de la información está bastante vapuleada. También, desde hace ya bastante tiempo, se ha puesto en crisis la ética periodística y los valores que implica comunicar con responsabilidad y garantizando el derecho a la comunicación, la libertad de expresión de las mujeres y de las personas con identidades de género diversa (13). En el siguiente apartado, exponemos los resultados del análisis de las fuentes informativas relevadas en tres periódicos locales con el fin de ejemplificar la discriminación de género como evidencia de la violencia simbólica que los medios ejercen cotidianamente.

\section{La perspectiva de género en el uso de fuentes}

Como hemos visto hasta aquí la ausencia de perspectiva de género en la producción periodística tiene múltiples causas y consecuencias que afectan tanto a las relaciones laborales como el producto del trabajo informativo. Un modo de reconocer su impacto en la tarea periodística es detenernos a observar el uso de las fuentes informativas como un indicador de la discriminación de género en las redacciones.

«Las fuentes informativas son un elemento clave del periodismo. Su capacidad para proporcionar datos sobre acontecimientos y sucesos de actualidad las sitúa como un componente imprescindible (...) la centralidad de las fuentes es tal que permite afirmar que sin ellas no hay periodismo» (Casero Ripollés y López Rabadán, 2013: 73) 
Al observar las fuentes como indicador de calidad por su capacidad para condicionar el resultado final de una noticia, el último informe GMMP (2015) señala que el $82 \%$ de portavoces y el $91 \%$ de expertos que aparecen en las noticias son varones. El informe sostiene que la diferencia de género en la selección de la fuente es extremadamente significativa, además de una tendencia observada desde el año 2000, por lo que concluye que la reducción de la brecha de género, también en el uso de fuentes, debería ser parte de una estrategia general que reúna la multiplicidad de condiciones que respalden los objetivos de la igualdad y pluralidad de voces en las noticias.

Para seleccionar la fuente, la lente de género en el periodismo no sólo se centra en los varones, sino que, hasta cierto punto, está sesgada a cierta masculinidad hegemónica. A la mayor parte de los sujetos, los portavoces y los expertos citados en las noticias -lo mismo mujeres que varones- se los describe como funcionarios públicos de alto nivel y políticos. La pauta se mantiene para los varones en todo tipo de funciones: el $12 \%$ de los varones que opinan con base en su experiencia personal, el 16\% de los testigos y el 10\% de los que ofrecen su opinión personal ostentan privilegios políticos y económicos (GMMP, 2015).

La UNESCO ha señalado la escasez de fuentes de información femenina como la principal causa del problema de la brecha de género en los medios de comunicación (Zapata, 2007) (14). Ciertamente, la gestión de las fuentes otorga visibilidad social a individuos y colectivos y a sus reivindicaciones y propuestas en la esfera pública.

«Por lo tanto, cuantas más visiones e intereses se expresan en las noticias, utilizando una mayor diversidad de actores sociales como fuentes, mayor pluralismo social reflejarán las noticias. Así el recurso a una polifonía de voces potencia la capacidad democratizadora del periodismo» (Casero Ripollés y López Rabadán, 2013: 77).

A su vez, el uso de las fuentes puede ser considerado como un criterio de calidad periodística. Para evaluarlo, en el siguiente apartado nos adentramos en su análisis, tomando todas las noticias publicadas, en una jornada informativa aleatoria.

\section{Análisis del uso de fuentes en periódicos locales}

Con el fin de indagar en torno a la segregación de las voces femeninas y de otras comunidades subalternas en las noticias desarrollamos este ejercicio práctico relevando las fuentes consultadas por los tres periódicos impresos (15) publicados en la ciudad de Rosario: La Capital, Rosario 12 y El Ciudadano. El corpus se configuró con la selección de un día al azar procurando que no coincidiera con efemérides vinculadas a las reivindicaciones del movimiento de mujeres o feministas (16).

Se relevaron todas las noticias publicadas el domingo, 20 de mayo de 2018, por ser el día de la semana de mayor tirada y circulación de los periódicos impresos locales. El criterio metodológico para analizar las fuentes contempló las siguientes variables:

1. Porcentaje de varones y mujeres consultados en cada diario

2. Ubicación de dichas fuentes en las secciones temáticas ofrecidas por cada diario

3. Distribución del porcentaje de género en las páginas $\mathrm{y}$

4. La relación entre las fuentes identificadas y el género de la autoría de la noticia.

Asimismo, distinguimos las fuentes no humanas (17) 


\section{II: Feminismo y medios de comunicación}

utilizadas y, también, la ausencia de fuentes en las noticias (no consta).

Respecto a la primera variable, en términos cuantitativos las voces de varones supera todas las categorías, representando el $56 \%$ del total de fuentes consultadas. Las voces femeninas representan el 17\%, mientras que las voces no humanas o las noticias con ausencia de fuentes superan, en conjunto, la cantidad de mujeres referenciadas como fuentes.

Cuadro 1: Presencia de mujeres y varones en las fuentes consultadas

\begin{tabular}{|c|c|c|c|c|c|}
\hline Diarios & MUJERES & & VARONES & $\begin{array}{c}\text { NO } \\
\text { HUMANAS }\end{array}$ & $\begin{array}{c}\text { NO } \\
\text { CONSTA }\end{array}$ \\
\hline La Capital & 10 & & 25 & 5 & 7 \\
\hline Rosario 12 & 5 & & 18 & 5 & 10 \\
\hline El Ciudadano & 7 & & 30 & 6 & 3 \\
\hline Total & 22 & $17 \%$ & 73 & 16 & 20 \\
\hline
\end{tabular}

Si nos detenemos a observar la segunda variable analizada que corresponde con la ubicación de dichas fuentes en las secciones temáticas ofrecidas por cada diario, tal como se muestra en el siguiente cuadro:

Cuadro 2: Porcentaje de género por temática de la sección

\begin{tabular}{|l|r|r|r|}
\hline Tema de la sección & MUJERES & VARONES & NO CONSTA \\
\hline Política/Economía & $2,6 \%$ & $15,8 \%$ & $81,6 \%$ \\
\hline Región/Mundo/Pais & $4,8 \%$ & $14,3 \%$ & $81,0 \%$ \\
\hline Opinión & $33,3 \%$ & $66,7 \%$ & $0,0 \%$ \\
\hline La Ciudad & $16,7 \%$ & $25,0 \%$ & $58,3 \%$ \\
\hline Información General & $14,3 \%$ & $14,3 \%$ & $71,4 \%$ \\
\hline \hline Policiales & $0,0 \%$ & $13,3 \%$ & $86,7 \%$ \\
\hline Deportes & $0,0 \%$ & $50,0 \%$ & $50,0 \%$ \\
\hline Espectáculos y Cultura & $11,1 \%$ & $33,3 \%$ & $55,6 \%$ \\
\hline Total & $8,20 \%$ & $21,31 \%$ & $70,49 \%$ \\
\hline
\end{tabular}


El cuadro refleja las voces más consultadas por sección temática, tomando el conjunto de periódicos analizados. Los datos ponen de manifiesto que cuando las fuentes son explicitadas, las opiniones de varones superan a las de las mujeres en todos los temas, a excepción de las noticias publicadas en la sección Información General donde muestran paridad. Asimismo, se destaca el alto porcentaje de fuentes no explicitadas en todos los diarios analizados.

Para analizar la distribución del porcentaje de género en las páginas de los tres diarios tuvimos en cuenta que las primeras páginas, al igual que la portada, incluyen la información relevante, según la lógica que estructura la diagramación del discurso informativo en su presentación impresa. Por ello establecimos como criterio de análisis la aparición de las fuentes distribuidas por género en las primeras 10 páginas, las segundas 10 páginas y las que se mencionan explícitamente después de la página número 20 , tal como se muestra a continuación:

Cuadro 3: Distribución de género por orden de páginas

\begin{tabular}{|l|c|c|c|} 
Orden & MUJERES & VARONES & NO CONSTA \\
\hline Primeras 10 & $8,16 \%$ & $24,49 \%$ & $67,35 \%$ \\
\hline Segundas 10 & $11,11 \%$ & $11,11 \%$ & $77,78 \%$ \\
\hline Después De La 20 & $3,57 \%$ & $32,14 \%$ & $64,29 \%$ \\
\hline Total & $8,20 \%$ & $21,31 \%$ & $70,49 \%$
\end{tabular}

En este cuadro se observan datos que complementan los resultados expuestos en el anterior. La mayor presencia de fuentes masculinas se ubica tanto en las 10 primeras páginas como en las ultimas, coincidiendo con las secciones temáticas de: Política/Economía, Región/Mundo/País, Opinión y La Ciudad, por un lado, y Policiales, Deportes, Espectáculos y Cultura, por el otro.

Por último, al relacionar las fuentes identificadas con el género de la autoría de cada noticia observamos que, en las noticias firmadas por mujeres se recurre, proporcionalmente, a más fuentes femeninas que en las noticias firmadas por varones, tal como se muestra en el siguiente cuadro:

Cuadro 4: Correspondencia entre género de autorías y género de fuentes citadas

\begin{tabular}{|l|r|r|r|r|}
\hline $\begin{array}{c}\text { Autoría } \\
\text { Fuent } \\
\mathrm{e}\end{array}$ & MUJERES & VARONES & NO CONSTA & TOTAL \\
\hline Mujer & $21,05 \%$ & $36,84 \%$ & $42,11 \%$ & $100.00 \%$ \\
\hline Varón & $9,09 \%$ & $31,17 \%$ & $57,14 \%$ & $100.00 \%$ \\
\hline No Consta & $0,00 \%$ & $9,09 \%$ & $90,91 \%$ & $100.00 \%$ \\
\hline
\end{tabular}


Esta última variable analizada, a su vez, nos permitió constatar, en primer lugar, que las noticias donde no consta firma profesional superan ampliamente a las noticas formadas. Y, en segundo lugar, que se corrobora lo problematizado en el apartado anterior respecto a que las mujeres periodistas son "la tropa" de las redacciones ya que al contabilizar el tipo de fuentes utilizadas en las noticias firmadas pudimos constatar que en el conjunto de los diarios analizados sólo el $22 \%$ de las autorías explicitadas corresponden a profesionales mujeres, mientras que el $78 \%$ restante a varones. De estos datos podemos inferir que el crecimiento de la participación de las mujeres en la profesión periodística en calidad de redactoras "no consta".

\section{A modo de cierre}

Hasta aquí procuramos recorrer los puntos problemáticos que señalamos al principio revisando aspectos vinculados a la discriminación de género en la profesión periodística, el problema de las violencias sexistas en todos los ámbitos y las luchas del movimiento para erradicarla. Asimismo, revisamos el problema de la ausencia de perspectiva de género en la producción informativa como indicador de la discriminación en las redacciones.

Claro está que el análisis aquí propuesto es apenas un recorrido por las particularidades que adopta esta problemática en la región, ya que entendemos que el uso de fuentes en el periodismo involucra toda una línea de investigación más amplia, pero no queríamos dejar de vincular los actuales debates del feminismo sobre la violencia simbólica que ejercen los medios como reflejo de las estructuras de poder en la sociedad. El uso de fuentes como mecanismo de legitimación del poder androcéntrico, es abordado por diversos autores, destacamos el aporte de Alsina (1993) quien plantea que "las fuentes que aparecen en los discursos informativos son importantes porque son las que se institucionalizan socialmente. Incluso cabría apuntar que son elementos esenciales para el estatuto veredictorio de estos discursos". Interpelar dicha función legitimadora resulta esencial desde la perspectiva de los estudios de género, ya que posibilita observar en qué medida la ausencia de fuentes feminizadas en la producción de noticias conlleva una deslegitimación de las voces de las mujeres y otros sectores subalternizados en la construcción de la ciudadanía plural.

La visibilización de estos fenómenos a partir de la popularización de las disputas protagonizadas por los feminismos (Rovetto y Figueroa, 2018), nos convoca como académicas, comunicadoras y militantes a una revisión de las prácticas profesionales del periodismo, para transformar no solo lo que dice (el contenido) sino también cómo lo dice y cómo se reproducen los mecanismos de desigualdades de género durante la producción de contenido.

Consideramos que una incorporación más extendida de este enfoque en materia de comunicación dependerá de la dinámica de las estructuras institucionales mismas que, como hemos visto, están organizadas desde una perspectiva masculina que afecta cada parte del proceso y también a cada participante del mismo. A su vez, su desarrollo efectivo también dependerá de la aplicación real los marcos jurídicos y legales existentes, pero más aún, de la disputa de poder político que el movimiento feminista materialice, accediendo a espacios y recursos materiales, así como de su creatividad para "comunicar" la necesaria transformación radical de nuestras sociedades. 


\section{1- La fuente de las desigualdades en las redacciones periodísticas. Voces feministas contra la discriminación y la violencia de género en los medios}

Cierto es que, a pesar de los avances en materia de regulación internacional, la mirada informativa permanece anquilosada en un enfoque androcéntrico, reducido a varones adultos que ocupan lugares de poder, de modo que la prensa resulta insensible a las transformaciones políticas y sociales de los últimos años, entre las que se destaca el avance de las mujeres y de otras identidades sexo-genéricas en todos los ámbitos. Estamos convencidas de que los papeles sociales que durante siglos se habían atribuido a varones y mujeres ya no sirven porque distorsionan la realidad amén de vulnerar los derechos del conjunto de la ciudadanía. La introducción de la perspectiva de género en la información puede ser una nueva manera de mirar, de interpretar la realidad con otros ojos, de hacer posible que aparezcan otras visiones del mundo y romper con las inercias profesionales, las rutinas establecidas y los dogmas no cuestionados. Como han señalado las perio-feministas de Pikara Magazine, hará falta ponerse masivamente las gafas violetas, esas que permiten reparar en las desigualdades de género como condicionantes de muchas realidades sobre las que informar. El periodismo sexista no puede ser bueno porque no atenta sólo contra los preceptos del feminismo sino también contra las reglas básicas del periodismo. $\mathrm{Y}$, volviendo al contexto de indagación, hará falta que los profesionales del periodismo se hagan cargo de lo que la Colectiva de Actrices señaló en el comunicado de prensa que acompañó la denuncia pública de Thelma Fardín:

«(...) deploramos que algunos medios intenten llevar la atención hacia el costado más morboso de los conflictos, mientras acallan las problemáticas laborales de fondo. Se vuelven cómplices. Le pedimos a la

\section{Monográfico}

prensa responsabilidad en el seguimiento de este tema y de otros similares»

Solo visibilizando los sentidos de la desigualdad, podemos enriquecer los medios de comunicación y las audiencias, aportando claves de análisis que nos ayuden a entender mejor cómo funciona el mundo. No podemos cumplir con ese objetivo si obviamos el impacto que tiene el sexismo sobre todo lo que nos rodea.

Notas

(1)El programa "Intrusos en el Espectáculo", conducido por Jorge Rial desde el año 2001, se emite por la señal América TV a primera hora de la tarde. Desde su inicio logró cautivar a una gran audiencia femenina con periodismo de espectáculo, centrado en la farándula o popularmente, denominado «programa de chimento». Para profundizar sobre las características y relevancia en materia de contenidos y consumo popular de este programa consultar los trabajos de Borda y Spataro (2018), De Tito (2018) Justo Von Lurzer (2017) y Spataro (2017).

(2) Promocionado como el \#MeToo argentino y con la consigna \#MiraComoNosPonemos, el 11 de diciembre de 2018, la colectiva de Actrices Argentinas organizó una conferencia de prensa donde leyeron un comunicado y pautaron intercambio con la prensa. $\mathrm{Al}$ respecto se pueden consultar notas y entrevistas publicadas en medios generalistas y especializados los días posteriores. En particular recomendamos los siguientes artículos que analizan en profundidad el fenómeno: http://cosecharoja.org/asi-se-teje-unared-feminista-internacional/; https://www.elciudadanoweb.com/mira-como-nos-pusimos/ 
(3) Son muchos los trabajos que han podido constatar esta evidencia, sólo para nombrar algunos de los estudios más relevantes que tomamos como referencia en nuestra línea de indagación, destacamos los realizados en España por López Díez (2005); Bach y otros (2000); Gallego y otros (2002; 2007); y por Chaher y Santoro (2007; 2010); Fernández Hasan (2011), entre otras a nivel local.

(4) En torno al problema de las desigualdades de género en el ejercicio de la profesión periodística mencionamos algunos de los trabajos de consulta o de autoría propia, centrados en indagar sobre quiénes construyen noticias y cómo lo hacen, analizando las dinámicas de relaciones en las organizaciones periodísticas, los perfiles profesionales y prácticas en estos particulares espacios de trabajo y producción: García Albi (2007); Papí Gálvez (2008) Rovetto (2013; 2017); Rovetto y Figueroa (2015); entre otras.

(5) Las periodistas paramos. Trabajadoras del periodismo y la comunicación nos sumamos a la huelga del 8M. Disponible en: https://lasperiodistasparamos. wordpress.com/

(6) La segunda Huelga Internacional de Mujeres se extendió por 70 países y 150 ciudades en el mundo: "Madrid será la tumba del machismo", exclamaron las españolas; "El lugar de la mujer es la resistencia "gritaron las turcas desafiando al Estado sirio; "Time's up" ("Se acabó el tiempo"), expresaron las norteamericanas; "Aborto legal ya - Basta de ajuste y precarización laboral”, se escuchó en las principales ciudades argentinas con las movilizaciones más grandes de los últimos años (García, 2018).

(7) Según un informe del CEPA, la brecha de ingresos entre varones y mujeres se mantiene entre el 20 y $30 \%$ desde hace más de una década. La brecha salarial consiste en un indicador económico y socio-ocupacio- nal complejo que no se reduce al incumplimiento del derecho constitucional "igual remuneración por igual tarea", sino que resulta de la comparación de masas salariales (cantidad de horas por salario). Por ejemplo, en Argentina la tasa de actividad femenina al primer trimestre de 2018 ascendió a 48,5\% mientras que la de los varones registró $69,5 \%$. La significativa diferencia entre ambas es del $20 \%$. Además del diferencial en horas trabajadas, la segregación ocupacional se considera por la división entre actividades feminizadas y actividades masculinizadas, donde las primeras perciben menores ingresos. La inserción ocupacional de gran parte de las mujeres tiene lugar en actividades consideradas feminizadas, asociadas a tareas del cuidado. Asimismo, en la ponderación de la brecha salarial se debe incluir que las mujeres padecen una doble jornada laboral (se estima que destinan 6 horas promedio por día a tareas domésticas), pero solo perciben ingresos por una jornada, la cual tiene - por otro lado- menos horas que la de los varones y por ende se traduce en la percepción de ingresos menores (Strada, 2018).

(8) Si bien, en los últimos años, la metáfora del techo de cristal ha tenido amplia difusión y adhesión para explicar los límites no escritos o invisibles en el avance social y político de las mujeres (López Díez, 2004), algunas autoras han procurado revisar este concepto, sosteniendo que se parte del error al pensar que mujeres y varones tienen idénticas posiciones de partida y que la metáfora singulariza uno de los obstáculos visibilizando otras dificultades que encuentran a lo largo de su vida laboral. Así, Eagly y Carli (2007) sostienen que más que una barrera con la que chocan y que frena en seco su ascenso profesional, se trata de un laberinto lleno de obstáculos del que es muy complejo salir.

(9) El estudio publicado, recientemente, por ONU 
Mujeres y Fundación Internacional de Mujeres Periodistas (IWF) indica que los hombres todavía ocupan el $73 \%$ de los altos cargos directivos en el sector de los medios de comunicación a nivel mundial. Disponible en: http://www.unwomen.org/es/news/stories

(10) En referencia a las etapas históricas del feminismo que se sistematizan por olas, ya que ante cada avance histórico de las mujeres y de las identidades feminizadas, se lanzan contraofensivas que quieren hacer retroceder los derechos conquistados. Lo que hoy se reconoce como la Cuarta Ola, con una fuerte impronta internacionalista, se caracteriza por altos grados de movilización social y articulación a nivel global -gracias a las tecnologías infocomunicacionales de las que han sabido apropiarse las colectivas feministas-, elevados niveles de transversalidad sectorial y generacional y una radicalidad en las demandas, acompañada de una mayor capacidad de incidencia política (Altamirano y otros, 2018).

(11) Siguiendo los lineamientos de la Convención Interamericana para Prevenir, Sancionar y Erradicar la Violencia hacia las Mujeres, conocida como Belém do Pará y aprobada por la Organización de Estados Americanos en 1994, las leyes de violencia llamadas "de segunda generación" que comenzaron a aprobarse en diversos países latinoamericanos, entrada la primera década del siglo XXI, son superadoras de las normas aprobadas en los años '90 que remiten a la violencia doméstica y/o familiar. Las nuevas leyes refieren a la violencia de género, tipifican sus modalidades en todos los ámbitos en los que se producen y la consideran como una violación de los derechos humanos de las mujeres teniendo en cuenta su diversidad.

(12) Por ejemplo, la Ley de Servicios de Comunicación Audiovisual fue modificada por el Decreto de Necesidad y Urgencia (DNU) 267/2015, firmado en diciembre de 2015, por el actual jefe del ejecutivo nacional, Mauricio Macri. Esta modificación ha implicado un retroceso en materia de derechos, interrumpiendo su efectiva aplicación, dejando sin efecto el desarrollo de medidas que puedan favorecer, entre otras cosas, la inclusión de la perspectiva de género en los medios de comunicación. Al mismo tiempo, el mencionado DNU, disolvió el Consejo Federal de Comunicación Audiovisual y el Consejo Federal de Tecnologías de las Telecomunicaciones, que incorporan la activa participación de representantes de los sindicatos, las universidades y las provincias.

(13) Ya el Informe MacBride, también conocido como "Voces Múltiples, Un Solo Mundo", publicado en 1980 por la UNESCO el Informe, advertía sobre las relaciones que se establecen entre los campos de la comunicación, las relaciones de poder y la democracia, proponiendo un nuevo equilibrio mundial en el ámbito de la información y de la comunicación para promover la paz y el desarrollo humano.

(14) De ahí que, los Objetivos de Desarrollo del Milenio presentados por la UNESCO se propusieran que el $30 \%$ de las fuentes de información de los artículos sean voces femeninas, animando a empresas y organizaciones periodísticas a sumarse a la iniciativa. Sin embargo, esta propuesta de cupo como medida afirmativa, hasta hoy no se ha cumplido (Chaparro, 2015).

(15) La decisión de analizar los diarios locales en su versión impresa remite a la estabilidad del discurso informativo en este tipo de soporte. Si bien todos los periódicos analizados tienen su versión digital, en ellas se pueden modificar los contenidos e introducir cambios a lo largo de la jornada informativa que 
pueden afectar el criterio de relevar "una foto fija", representativa del discurso informativo.

(16) Nos referimos a las fechas que coinciden con las efemérides conmemoradas por las organizaciones feministas o de la diversidad sexual que pueden motivar mayores niveles de presencia de mujeres o personas feminizadas consultadas, distorsionando los parámetros habituales e invalidando el ejercicio analítico propuesto.

(17) Respecto a esta categoría se recomienda consultar la Guía para Humanizar la Información (Moreno Sardà, Rovetto y Buitrago, 2007). Allí desarrollamos el Test de análisis y crítica al androcentrismo en el discurso informativo, donde relevamos protagonistas, fuentes y autorías y los contextos espacio-temporales de las noticias. La categoría No Humana (aplicada a protagonistas y fuentes) surgió al interrogarnos sobre de quién hablan las noticias, cuando detectamos la presencia creciente de fenómenos que englobamos como no humanos, para agrupar una diversidad de instituciones, entidades, seres animados e inanimados, acciones, datos..., que cada vez más aparecen como los protagonistas activos y los sujetos agentes de las acciones noticiables. Un grupo de protagonistas heterogéneo en el que predominan una serie de instituciones, entidades y corporaciones desde las que se ejerce el poder en distintas esferas, y también datos abstractos, estadísticas, acciones diversas que a menudo, en lugar de enriquecer la información, suplantan a los seres humanos responsables y presentan determinadas situaciones y actuaciones como si fueran asépticas; en definitiva, hacen la información más opaca, más alejada de la realidad, más deshumanizada.
Referencias

Alsina, M. R. (1992). La construcción de la noticia. Barcelona: Paidós.

Bach, M., Altés, E. y otros (2000). El sexo de la noticia. Reflexiones sobre el género en la información y recomendaciones de estilo. Barcelona: Icaria.

Balseiro Expósito, A. (2008) Terrorismo de género en los medios de comunicación: la imagen de la violencia frente a la violencia de la imagen. Madrid: Universidad Carlos III de Madrid.

Borda, L. y Spataro, C. (2018) El chisme menos pensado: el debate sobre aborto. En Intrusos en el espectáculo. Sociales en debate $\mathrm{N}^{\circ} 14$. FCSoc, UBA. Disponible en: https://publicaciones.sociales.uba.ar/ index.php/socialesendebate/article/view/3353

Casero-Ripollés, A. y Rabadán, P. L. (2013). La Gestión de fuentes informativas como criterio de calidad profesional. En La calidad periodística: teorías, investigaciones y sugerencias profesionales. Castelló de la Plana: Universitat Jaume I, pp. 73-89.

Chaher, S. y Santoro, S. (2007). Las palabras tienen sexo. Introducción a un periodismo con perspectiva de género. Buenos Aires: Artemisa comunicaciones.

Chaher, S. y Santoro, S. (2010). Las palabras tienen sexo II. Herramientas para un periodismo de género. Buenos Aires: Artemisa comunicaciones.

Chaparro, L. (2015): "Mujeres ocultas bajo un 'techo de papel’: ¿Por qué ellas están infrarrepresentadas en los medios?" Disponible en: http://www.publico.es/ sociedad/mujeres-ocultas-techo-papel.html

de Titto, J. (2018). Crear un nuevo mundo también es narrarlo. En VV. AA. La cuarta ola feminista. Buenos Aires: Oleada / MalaJunta.

Eagly, A. H. y Carli, L. (2007). Through the labyrinth: 
The truth about how women become leaders. Cambridge: Harvard Business Press.

Femenías, M. L. (comp.) (2002). Perfiles del feminismo iberoamericano. Buenos Aires: Catálogos.

Gallego Ayala, J. y Altés, E., (2002). La prensa por dentro: producción informativa y transmisión de estereotipos de género. Barcelona: Libros de la Frontera. Gallego Ayala, J. y otros (2007). Buenas prácticas periodísticas desde la perspectiva de género. Madrid: AMECO, Asociación Española de Mujeres Profesionales de los Medios de Comunicación.

García Albi, I. (2007). Nosotras que contamos: mujeres periodistas en España. Madrid: Plaza \& Janes.

García, M. P. (2018). Una ola feminista recorre el mundo. En VV. AA. La cuarta ola feminista. Buenos Aires: Oleada / MalaJunta.

GMMP (2015). Proyecto de Monitoreo Global de Medios. ¿Quién figura en las noticias? Desigualdad de Género en las Noticias 1995-2015. Canadá: WACC.

Henry, L. (2013). Fragmentación y precarización laboral en la prensa escrita. Los desafios para la representación y la organización colectiva de los periodistas en un entorno productivo flexibilizado. La revitalización sindical en Argentina: alcances y perspectivas. Buenos Aires: Universidad Nacional de la Matanza, Editorial Prometeo.

Hirata, H. (1997). La división sexual del trabajo: permanencia y cambio. Buenos Aires: Centro de Estudios de la Mujer de Chile

Justo Von Lurzer, C. (2017). Esto le puede servir a alguien. Demandas de derechos en el espectáculo televisivo contemporáneo en Argentina. En Revista Estudos de Comunicação. Disponible en: http://ojs. labcom-ifp.ubi.pt/index.php/ec/article/view/279. // Lagarde, M. (2015). Claves feministas para mis so- cias de la vida. Buenos Aires: Batalla de Ideas.

Lakoff, G. (2006). No pienses en un elefante. Madrid: Editorial Complutense.

López Díez, P. (2004). El techo de cristal también existe en los medios. Disponible en: http://mujeres. usal.es/data/core/archivos/congresoint.doc,2004.

López Díez, P. (2005). Representación de género en los informativos de radio y televisión. Segundo Informe Representación de Género en los Informativos de Radio y Televisión-Instituto de la Mujer-Ministerio de Trabajo y Asuntos Sociales. Madrid: Instituto Oficial de Radio y Televisión, RTVE.

Moreno Sardà, A. (1998). La mirada informativa. Barcelona: Bosch.

Moreno Sardà, A., Rovetto, F. y Buitrago, A. (2007). ¿De quién hablan las noticias? Guía para humanizar la información. Barcelona: Icaria.

ONU (1995). La mujer y los medios de difusión. En Declaración de la Plataforma de Acción de Beijing, Cap. III, Ap. J (pp. 171-177). Disponible en:

http://www.unwomen.org/ /media/headquarters/attachments/sections/csw/bpa_s_final_web.pdf

Papí Gálvez, N. (2008). El género entre bastidores. El caso de las periodistas de la Comunidad Valenciana. Alicante: Universidad de Alicante.

Pateman, C. (1995). El contrato sexual. México: Anthropos.

Rastrollo, M. A. y Gómez Torralbo, R. (2006). Informe sobre la situación laboral de las mujeres en los medios. En Farías Battle, P. (dir.) Informe sobre la Profesión Periodística 2006. Madrid: APM.

Rojo Brizuela, S. y Tumini, L. (2008). Inequidades de género en el mercado de trabajo de la Argentina: las brechas salariales. En Revista de Trabajo, 4(6).

Rovetto, F. (2013). Percepciones sobre desigualdades 


\section{II: Feminismo y medios de comunicación}

de género en el trabajo periodístico. Global Media Journal México. Monterrey: Centro de Investigación en Comunicación e Información, pp. 54-73.

Rovetto, F. (2015). Violencia contra las mujeres, comunicación visual y acción política en \#Ni Una Menos y \#Vivas Nos Queremos. En Contratexto, 1. (23); 13-34.

Rovetto, F. y Figueroa, L. (2015). "La cocina del trabajo periodístico. Desigualdades de género y distribución de poder en las redacciones informativas de la provincia de Santa Fe". Question. Revista Especializada en Periodismo y Comunicación. 1 (48); 494-509. Rovetto, F. (2017). "Igualdad de oportunidades y trabajo periodístico. Retos, retóricas y obstáculos". En Revista Internacional de Comunicación y Desarrollo. 7 (2); 35-46.

Rovetto, F. y Figueroa L. (2018). "Perio-feminismo desde adentro. Desigualdades de género en los medios.". En Revista Con X, 4. Disponible en: https:// perio.unlp.edu.ar/ojs/index.php/conequis/article/ view/5006

Segato, R. (2013). Historizando el patriarcado- Una trayectoria personal en el campo de los estudios de género. Curso virtual (Asociación Civil Comunicación para la Igualdad). Disponible en: http://www.comunicarigualdad.com.ar/curso-virtual-rita-segato-recorresu-trayectoria-en-estudios-e-gfebrero/
Spataro, C. (2018). Abajo el feministómetro, Revista Bordes (UNPAZ). Disponible en: http://revistabordes. com.ar/abajo-el-feministometro

Strada, J. (2018). Trabajo no remunerado, segregación ocupacional y políticas de ajuste: los ejes ausentes en el proyecto oficial de Equidad de Género. Centro de Economía Política Argentina (CEPA). Disponible en: http://centrocepa.com.ar/informes/108-trabajo-noremunerado-segregacion-ocupacional-y-politicas-deajuste-los-ejes-ausentes-en-el-proyecto-oficial-deequidad-de-genero.html

Wainerman, C. (1996). ¿Segregación o discriminación? El mito de la igualdad de oportunidades. En Boletín Informativo Techint, 285, pp. 59-75.

Zapata, D. (2007). Transversalizando la perspectiva de género en los objetivos de desarrollo del Milenio. Santiago de Chile: CEPAL. 